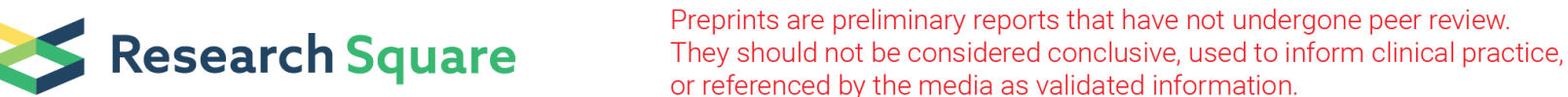

\section{ALK Rearrangement-Associated Renal Cell Carcinoma Morphologically Mimicking Mucinous Tubular And Spindle Cell Carcinoma: A Case Report}

Keita Kai ( $\sim$ kaikeit@cc.saga-u.ac.jp )

Saga University Hospital https://orcid.org/0000-0003-1553-2598

Shohei Tobu

Saga University: Saga Daigaku

Shinichi Kido

Saga University: Saga Daigaku

Shuji Mikami

National Hospital organization Saitama Hospital

Kengo Takeuchi

The Cancer Institute Japanese Foundation of Cancer Research

Akito Dobashi

The Cancer institution Japanese Foundation of Cancer Research

Yuki Togashi

The Cancer Institution Japan Foundation for Cancer Research

Mitsuru Noguchi

Saga University: Saga Daigaku

Shinichi Aishima

Saga University: Saga Daigaku

\section{Case Report}

Keywords: renal cell carcinoma, anaplastic lymphoma kinase, mucinous tubular and spindle cell carcinoma, case report

Posted Date: September 8th, 2021

DOI: https://doi.org/10.21203/rs.3.rs-812298/v1

License: (c) (1) This work is licensed under a Creative Commons Attribution 4.0 International License.

Read Full License 


\section{Abstract}

Background: Anaplastic lymphoma kinase rearrangement-associated renal cell carcinoma (ALK-RCC) is a very rare tumor and no single case report of ALK-RCC that mimics mucinous tubular and spindle cell carcinoma (MTSCC) has been reported.

Case presentation: A 42-year-old Japanese woman was admitted to our hospital for the treatment of a left renal tumor measuring approximately $5 \times 4 \mathrm{~cm}$ in diameter. She underwent a laparoscopic left nephrectomy. Histologically, the tumor formed tubular or focally papillary structures with a small amount of spindle-shaped tumor cells against the background of prominent extracellular mucin. Although the tumor cells were negative for immunohistochemistry (IHC) of alpha-methylacyl-CoA racemase (AMACR) and lymph node metastasis was presented (these are atypical findings for MTSCC), we initially diagnosed the tumor as MTSCC based on its morphological characteristics with mucin deposition. However, an additional IHC analysis revealed that the tumor cells were diffusely positive for ALK-IHC. In addition, TPM3 exon 8 - ALK exon 20 fusion gene was detected by RNA sequencing. The tumor was thus correctly diagnosed as ALK rearrangement-associated renal cell carcinoma (ALK-RCC).

Conclusions: Since the use of molecular targeted therapy with an ALK inhibitor for ALK-RCC is promising, the correct pathological diagnosis of ALK-RCC is quite important. We strongly recommend that ALK-IHC be routinely performed for renal tumors with negative AMACR staining that mimic MTSCC.

\section{Background}

Anaplastic lymphoma kinase rearrangement-associated renal cell carcinoma (ALK-RCC) is a very rare tumor that accounts for $<1 \%$ of all renal neoplasms [1]. Although ALK-RCC is currently considered an "emerging/provisional" type of renal cell carcinoma in the 2016 World Health Organization classification [2], the Genitourinary Pathology Society recently proposed categorizing ALK-RCC as a "novel entity" based on an accumulation of studies [3]. Because of its heterogeneous and diverse morphologies, the differential diagnosis of ALK-RCC varies widely, involving renal medullary carcinoma, collecting duct carcinoma, papillary RCC, MiT family translocation RCC, clear cell RCC with rhabdoid features, metanephric adenoma, thyroid-like follicular RCC, and mucinous tubular and spindle-cell carcinoma (MTSCC) [4].

We present a didactic case of ALK-RCC which was initially diagnosed as MTSCC. This case taught us the importance of considering the possibility of ALK-RCC in the pathological diagnosis of unusual renal tumors.

\section{Case Presentation}

\section{Clinical summary}


A 42-year-old Japanese woman was admitted to our hospital for the treatment of a left renal tumor that had been detected by a medical checkup. She was neither a habitual drinker nor a smoker. She had no remarkable medical or family history. The physical examination on admission was unremarkable. Laboratory hematologic, serological, and coagulation tests on admission revealed no abnormalities. Abdominal computed tomography and magnetic resonance imaging revealed a well-circumscribed solid tumor measuring approx. $5.4 \mathrm{~cm}$ in diameter at the left kidney. The tumor was hypovascular and protruded toward the renal pelvis. Radiologically, papillary RCC or chromophobe RCC were considered as differential diagnoses. A needle biopsy was performed, and the pathological report of biopsy specimens was "unclassified mucin-producing renal cell carcinoma."

The patient underwent a laparoscopic left nephrectomy. Intraoperatively, one swelling lymph node was identified at the hilum of the left kidney, and it was surgically dissected. The postoperative course was uneventful, and the patient was discharged from the hospital on the sixth postoperative day. She was periodically followed without adjuvant therapy, and no sign of recurrence had been detected at the time of this writing (16 months after the surgery).

\section{Pathological findings of resected specimens}

The cut section of the tumor (Fig. 1) revealed a well-circumscribed whitish solid tumor showing expansive growth. Histologically, tubular or focally papillary structures composed of tumor cells with eosinophilic cytoplasm and small-round nuclei were observed, and a small amount of spindle-shaped tumor cells and its transition figures were intermingled (Fig. 2). Prominent extracellular mucin deposition highlighted by alcian blue staining was observed (Fig. 3a).

Immunohistochemically, the tumor cells were positive for cytokeratin (CK) 7 (clone OV-TL 12/30, ready to use; Agilent Technologies, Santa Clara, CA) (Fig. 3b), paired box gene (PAX) 8 (clone MRQ-50, ready to use; Roche, Mannheim, Germany), and vimentin but negative for alpha-methylacyl-CoA racemase (AMACR) (clone 13H4, ready to use; Agilent Technologies) (Fig. 3c) and CD10 (clone SP67, ready to use; Roche). Lymph node metastasis was observed at one of the dissected lymph nodes. Although the lymph node metastasis and the negativity of AMACR are not typical findings of MTSCC, we initially diagnosed this tumor as MTSCC based on its morphological characteristics with mucin deposition.

We presented this case as an MTSCC at the 380th Kyushu-Okinawa slide conference (March 2021) held by the Kyushu-Okinawa Division of Japanese Society of Pathology. At that conference, some participants pointed out the possibility of the tumor being ALK-RCC. Following their comments, we performed an additional immunohistochemistry (IHC) analysis of the tumor by using ALK (D5F3) (clone D5F3, ready to use; Roche), and the tumor showed diffuse positivity at the cytoplasm and diffuse and strong positivity at the cell membrane (Fig. 3d). In addition, TPM3 exon 8 - ALK exon 20 fusion gene was detected by RNA sequencing. We thus finally reached the correct diagnosis of ALK-RCC. 


\section{Discussion And Conclusions}

RCC with rearrangement of ALK gene was first described by Debelenko et al. in 2011 [5]. Their report described a pediatric case with sickle cell trait, and the fusion partner of ALK gene was VCL gene. The first adult cases of ALK-RCC without a genetic background were reported in 2012 by Sugawara et al. [6]. They screened 355 RCC cases by ALK-IHC (using an intercalated antibody-enhanced polymer method) and identified two ALK-RCC cases which were confirmed to have TPM3-ALK and EML4-ALK fusion by fluorescence in situ hybridization assays.

Although ALK-RCC is extremely rare, its pathological features have been gradually revealed by the accumulation of case reports and studies [7]. ALK-RCC usually macroscopically presents a well- or illdemarcated solid tumor, but cystic changes or a cystic lesion have also been reported $[4,8,9]$. The histological morphology of ALK-RCC is highly variable and heterogeneous, not only between cases, but also within individual cases [4]. Most ALK-RCC cases have exhibited mixed architectural patterns, including papillary, tubular, trabecular, tubulocystic, and solid patterns. The tumor cells typically showed eosinophilic cytoplasm and various degrees of mucin deposition $[4,7]$.

ALK-RCCs usually express PAX 8, CK7, and vimentin, and no specific IHC marker other than ALK-IHC has been reported [4]. It has been suggested that a strong membranous stain is characteristic of TPM3-ALK fusion [6], and our patient's case also showed strong membranous positivity for ALK-IHC, supporting that suggestion. The reported fusion partners to ALK gene include VCL, TPM3, EML4, HOOK1, and STRN gene [7]. Recently, PLEKHA7, CLIP1, KIF5B and KIAA1217 were reported as fusion partners of ALK gene [4, 10].

As described in the Introduction, the differential diagnosis of ALK-RCC varies widely, and ALK-RCC may mimic various renal tumors. As ALK-RCC may show mucin deposition and a tubular structure, the importance of the differential diagnosis of MTSCC has been noted [7]. However, to the best of our knowledge, no single case report of ALK-RCC that mimics MTSCC has been reported. Only one multiinstitutional study of 12 cases described an ALK-RCC case morphologically resembling MTSCC which showed immunoreactivity compatible with MTSCC, i.e., positivity for PAX8, CK7, CD10, AMACR, and vimentin [4].

MTSCC is also a relatively rare epithelial neoplasm of low malignant potential with characteristic histologic features [11]. Although it was originally described as a tumor arising from cells of the loop of Henle or the collecting duct, the expression of CK7 and AMACR suggested its proximal nephron origin and its close resemblance to papillary RCC [12]. In the present case, although the findings of a lack of AMACR expression and lymph node metastasis were not typical findings of MTSCC, we initially considered MTSCC based on reports of rare cases of MTSCC that lacked AMACR expression, lymph node metastasis, and distant metastases $[10,13]$. Considering the above-mentioned ALK-RCC case morphologically resembling MTSCC with AMACR expression, we strongly recommend an analysis of ALK expression by IHC when diagnosing a renal tumor that mimics MTSCC. 
In conclusion, we have described a case of ALK-RCC which morphologically mimicked MTSCC. Since the use of molecular targeted therapy with an ALK inhibitor for cases of ALK-RCC is promising [6], the correct pathological diagnosis of ALK-RCC is quite important. In light of the lesson learned in the present case, we strongly recommend the routine performance of ALK-IHC in the pathological diagnosis of MTSCC.

\section{Abbreviations}

ALK-RCC: Anaplastic lymphoma kinase rearrangement-associated renal cell carcinoma; MTSCC: mucinous tubular and spindle-cell carcinoma; CK: cytokeratin; PAX 8: paired box gene 8; AMACR: alphamethylacyl-CoA racemase; IHC: immunohistochemistry

\section{Declarations}

\section{Acknowledgements}

We thank Dr. Hiroyuki Hayashi (Department of Pathology, Fukuoka University) and Dr. Daisuke Kiyozawa (Department of Pathology, Kyushu University), for their valuable comments regarding the pathological diagnosis. This case was presented and discussed at the 380th Kyushu-Okinawa slide conference. We are also grateful to the conference participants for their valuable comments and discussion.

\section{Author Contributions}

K.K. is the main author of this article. S.T., and M.N. treated patient. K.K., S.K., S.M., K.T., A.D. Y.T., and S.A. contributed to pathological diagnosis. All authors contributed to the editing of the submitted manuscript. All authors read and approved the final draft of the manuscript.

\section{Funding}

Not applicable.

\section{Availability of data and materials}

Data are available on reasonable request from the corresponding author due to privacy or other restrictions.

\section{Ethics approval and consent to participate}

As this is a case report, the Ethics Committee of the Faculty of Medicine at Saga University was not required the specific approval.

\section{Consent for publication}

We have obtained the patients' consent for publication and there is no personal information of the patients in our case report. 


\section{Competing interests}

The authors declare that they have no competing interests.

\section{Author details}

${ }^{1}$ Department of Pathology, Saga University Hospital, Saga, Japan, ${ }^{2}$ Department of Urology, Saga University Faculty of Medicine, Saga, Japan, ${ }^{3}$ Department of Pathology \& Microbiology, Saga University Faculty of Medicine, Saga, Japan, ${ }^{4}$ Department of Pathology, National Hospital Organization Saitama Hospital, Saitama, Japan, ${ }^{5}$ Division of Pathology, The Cancer Institute, Japanese Foundation for Cancer Research, Tokyo, Japan, ${ }^{6}$ Department of Pathology, The Cancer Institute Hospital, Japanese Foundation for Cancer Research, Tokyo, Japan, ${ }^{7}$ Pathology Project for Molecular Targets, The Cancer Institute, Japanese Foundation for Cancer Research, Tokyo, Japan

\section{References}

1. Kuroda N, Sugawara E, Kusano H, Yuba Y, Yorita K, Takeuchi K. A review of ALK-rearranged renal cell carcinomas with a focus on clinical and pathobiological aspects. Pol J Pathol. 2018;69:109-13.

2. Moch H, Humphrey PA, Ulbright TM, Reuter VE. WHO classification of tumours of the urinary system and male genital organs. 4th ed. Lyon: International Agency for Research on Cancer; 2016. pp. 16-7.

3. Trpkov K, Williamson SR, Gill AJ, Adeniran AJ, Agaimy A, Alaghehbandan R, et al. Novel, emerging and provisional renal entities: The Genitourinary Pathology Society (GUPS) update on renal neoplasia. Mod Pathol. 2021;34:1167-84.

4. Kuroda N, Trpkov K, Gao Y, Tretiakova M, Liu YJ, Ulamec M, et al. ALK rearranged renal cell carcinoma (ALK-RCC): A multi-institutional study of twelve cases with identification of novel partner genes CLIP1, KIF5B and KIAA1217. Mod Pathol. 2020;33:2564-79.

5. Debelenko LV, Raimondi SC, Daw N, Shivakumar BR, Huang D, Nelson M, et al. Renal cell carcinoma with novel VCL-ALK fusion: New representative of ALK-associated tumor spectrum. Mod Pathol. 2011;24:430-42.

6. Sugawara E, Togashi Y, Kuroda N, Sakata S, Hatano S, Asaka R, et al. Identification of anaplastic lymphoma kinase fusions in renal cancer: Large-scale immunohistochemical screening by the intercalated antibody-enhanced polymer method. Cancer. 2012;118:4427-36.

7. Kuroda N, Sugawara E, Kusano H, Yuba Y, Yorita K, Takeuchi K. A review of ALK-rearranged renal cell carcinomas with a focus on clinical and pathobiological aspects. Pol J Pathol. 2018;69:109-13.

8. Yang J, Dong L, Du H, Li XB, Liang YX, Liu GR. ALK-TPM3 rearrangement in adult renal cell carcinoma: A case report and literature review. Diagn Pathol. 2019;14:112.

9. Oyama Y, Nishida H, Kusaba T, Kadowaki H, Arakane M, Daa T, et al. A case of anaplastic lymphoma kinase-positive renal cell carcinoma coincident with Hodgkin lymphoma. Pathol Int. 2017;67:626-31. 
10. Hang JF, Chung HJ, Pan CC. ALK-rearranged renal cell carcinoma with a novel PLEKHA7-ALK translocation and metanephric adenoma-like morphology. Virchows Arch. 2020;476:921-9.

11. Nathany S, Monappa V. Mucinous tubular and spindle cell carcinoma: A review of histopathology and clinical and prognostic implications. Arch Pathol Lab Med. 2020;144:115-8.

12. Shen SS, Ro JY, Tamboli P, Truong LD, Zhai Q, Jung SJ, et al. Mucinous tubular and spindle cell carcinoma of kidney is probably a variant of papillary renal cell carcinoma with spindle cell features. Ann Diagn Pathol. 2007;11:13-21.

13. Paner GP, Srigley JR, Radhakrishnan A, Cohen C, Skinnider BF, Tickoo SK, et al. Immunohistochemical analysis of mucinous tubular and spindle cell carcinoma and papillary renal cell carcinoma of the kidney: Significant immunophenotypic overlap warrants diagnostic caution. Am J Surg Pathol. 2006;30:13-9.

\section{Figures}

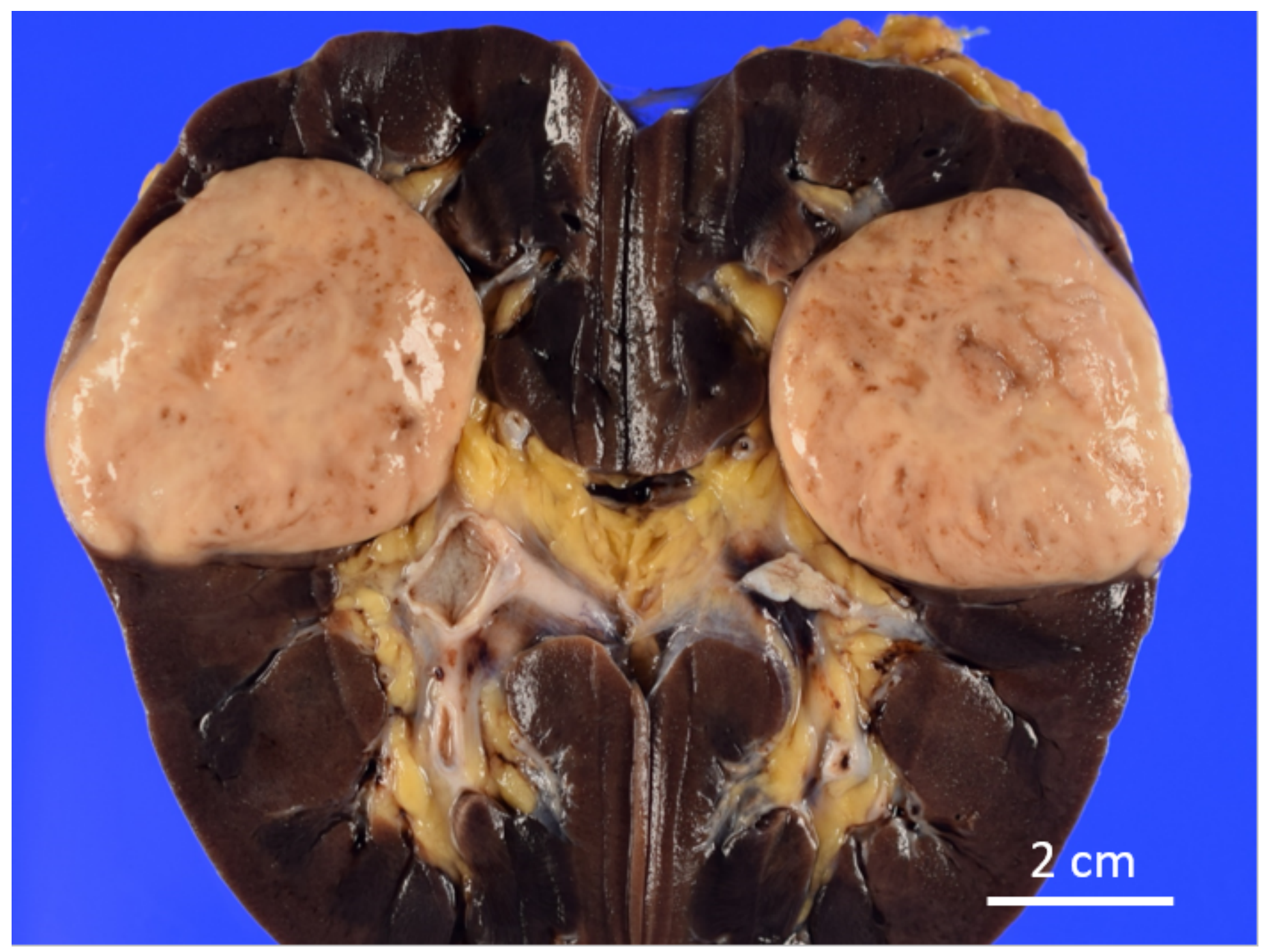

\section{Figure 1}


Gross appearance of the tumor. The tumor was well-circumscribed, whitish, and solid, and it showed expansive growth.
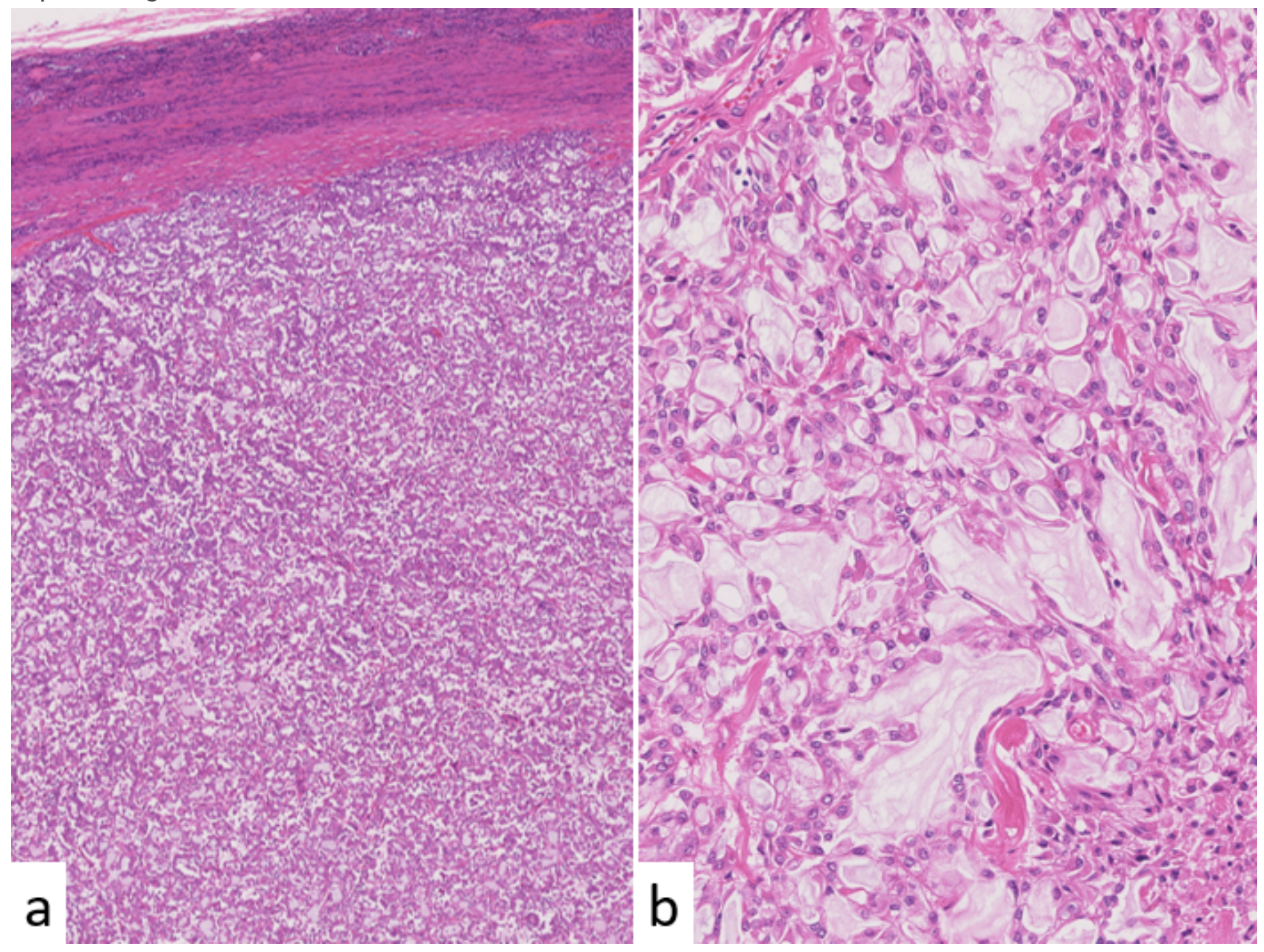

Figure 2

Histological appearance. a: Low magnification $(\times 50)$. The tumor showed expansive growth and formed tubular or papillary structures. b: High magnification $(\times 200)$. The tumor cells had eosinophilic cytoplasm and small-round nuclei. A small amount of spindle-shaped tumor cells and prominent extracellular mucin were observed. 

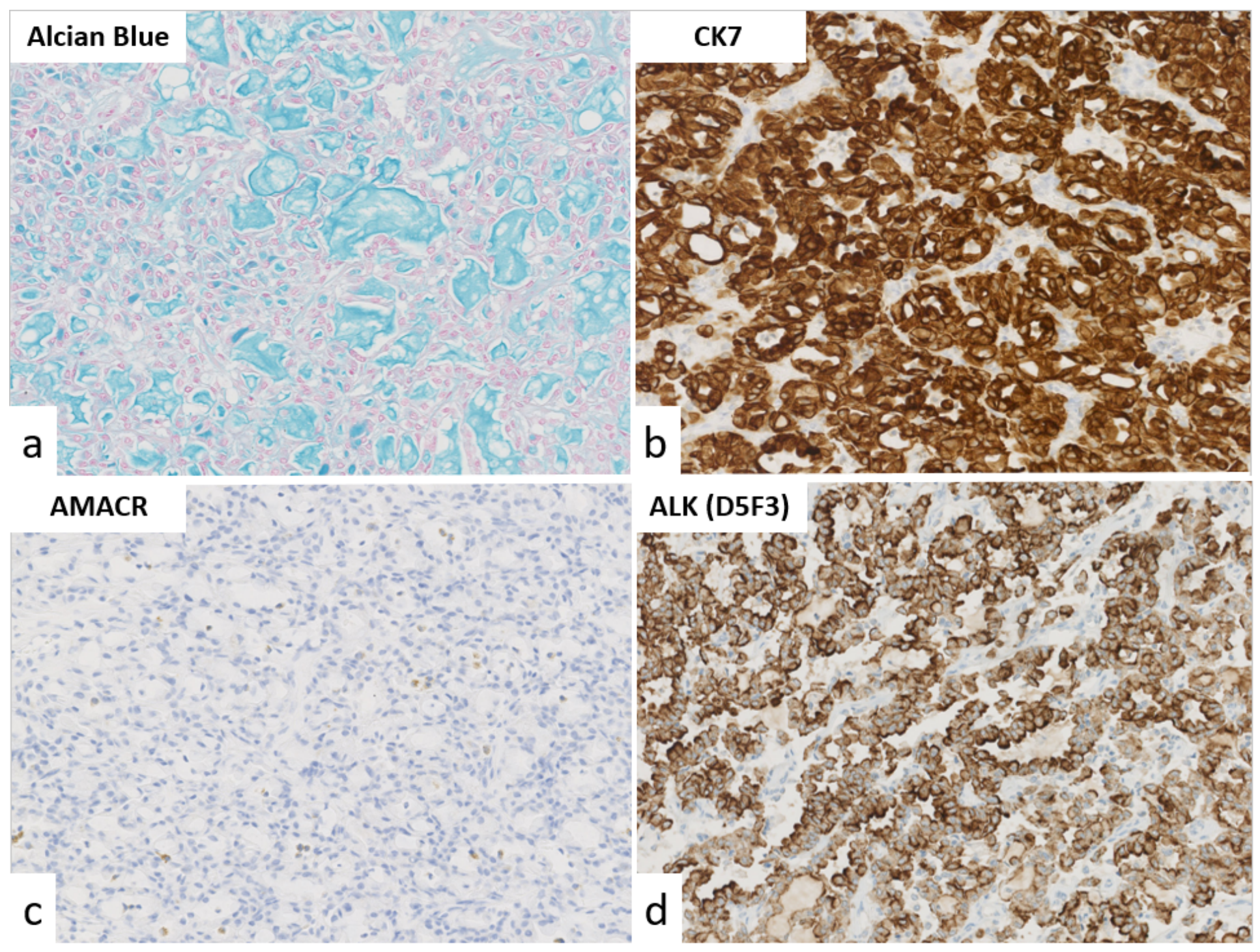

\section{Figure 3}

(a) Extracellular mucin deposition is highlighted by alcian blue staining). IHC results for CK7 (b), AMACR (c), and ALK(D5F3) (d). The magnification of all four slides is $\times 200$. The tumor showed diffuse positivity at the cytoplasm and diffuse and strong positivity at the cell membrane.

\section{Supplementary Files}

This is a list of supplementary files associated with this preprint. Click to download.

- CAREchecklistEnglish2013.pdf 